REZENSIONEN

\section{Das Nichteinhaltungsverfahren des Kyoto-Protokolls}

\author{
Chr. Holtwisch: Das Nichteinhaltungs- \\ verfahren des Kyoto-Protokolls. Entste- \\ hung - Gestalt - Wirkung. Berlin: Dun- \\ cker\&Humblot, 2006, 382 S., ISBN 3- \\ 428-12215-1, € 98
}

\section{Rezension von Gerhard Sardemann, ITAS}

Das Buch „Das Nichteinhaltungsverfahren des Kyoto-Protokolls" beruht auf dem Text der Dissertation des Autors im Fachbereich Rechtswissenschaft der Johann-WolfgangGoethe Universität Frankfurt, die im Jahr 2006 angenommen wurde. Vorangestellt wurden Geleitworte von Klaus Töpfer, der auf die vielen - mangels eines funktionierenden Nichteinhaltungsverfahrens „zahnlosen“ - Verträge und damit auf die große Relevanz des Themas hinweist, sowie von Hanns-Ferdinand Müller, Vorstandsmitglied der RWE AG, die sich mit einem Druckkostenzuschuss an der Veröffentlichung beteiligt hat.

Der Rezensent gibt freimütig zu, als Meteorologe mit den rechtswissenschaftlichen Feinheiten, insbesondere was die völkerrechtlichen Einhaltungstheorien angeht, nicht vertraut zu sein. Andererseits hatte er als Mitarbeiter der „Informationsstelle Umweltforschung“ (ITAS/ BMBF) Gelegenheit, den Verhandlungsprozess zur Klimarahmenkonvention seit den Sitzungen des Intergovernmental Negotiating Committee (INC) Anfang der 1990er Jahre über die erste Vertragsstaatenkonferenz in Berlin bis hin zur Konferenz in Kyoto im Auftrag des Bundesministeriums für Bildung und Forschung (BMBF) mitzuverfolgen und beschäftigt sich auch aktuell mit den verschiedenen Mechanismen des KyotoProtokolls. Zudem ist die hier besprochene Arbeit nach dem vom Verlag verteilten Flyer „nicht nur ein Verfahrenskommentar, sondern bietet auch eine einhaltungsbezogene Einführung in das Kyoto-Protokoll" und es wird aus dem Geleitwort Töpfers zitiert, die Arbeit sei „lesenswert sowohl für politische Entscheidungsträger, für Praktiker der Umsetzung, aber auch für Wissenschaftler".

Bevor nun auf den Inhalt des Buches eingegangen werden soll, ist dem Verlag für die Ausstattung, insbesondere für die Bindung in Fadenheftung, ein Lob auszusprechen. Dadurch wird das Werk auch bei häufiger Nutzung, die man, soviel sei vorab schon verraten, durchaus empfehlen kann, womöglich länger Bestand haben als das Kyoto-Protokoll selbst.

\section{Der Sach- und Rechtsrahmen des Nichteinhaltungsverfahrens}

Der Rechtsrahmen des Nichteinhaltungsverfahrens - so heißt es im ersten Kapitel - diene dazu, dem Ziel der Klimarahmenkonvention näher zu kommen, „die Treibhausgaskonzentrationen in der Atmosphäre auf einem Niveau zu stabilisieren, dass eine gefährliche anthropogene Störung des Klimasystems verhindert wird" (S. 28). Bevor man weiter liest, fragt man sich unwillkürlich, ob das Kyoto-Protokoll, zu dem das Nichteinhaltungsverfahren gehört, prinzipiell dazu geeignet ist, die Treibhausgaskonzentrationen in der Atmosphäre zu stabilisieren. Bislang haben sich nur die Industrieländer zu moderaten Reduktionen ihrer Emissionen verpflichtet; die USA machen gar nicht mit und haben außerhalb des UN-Rahmens mit der „Asia-Pacific Partnership on Clean Development and Climate" ein Konkurrenzunternehmen zum Kyoto-Protokoll aufgemacht.

Der Club der mehr oder weniger ,Willigen", der sich nun dem Regelwerk des KyotoProtokolls unterworfen hat, kommt einem manchmal so vor wie die von Loriot so vorzüglich karikierte Skatrunde, in der zwei Mitspieler ihrem dritten Mann jeden Regelverstoß durchgehen lassen müssen, um überhaupt weiterspielen zu können - bis dieser dann endgültig auch seine eigenen Karten zerstört. In trockneren Worten wird diese Problematik, insbesondere die eines möglichen Legitimationsverlustes des Kyoto-Protokolls und damit auch seines Nichteinhaltungsverfahrens, als Resultat der Klimaschutzalternativen der USA natürlich auch vom Autor thematisiert (S. 69 ff.), der übrigens hierzu inzwischen eine weitere Arbeit verfasst hat (Holtwisch 2007). 
Aber zurück zum Sachrahmen: Es geht darum, herauszufinden, wie das Klimasystem funktioniert, wie sich die Aktivitäten des Menschen darauf auswirken, was gefährliche Störungen des Klimasystems sind und wie sie sich vermeiden lassen. Mit diesen Fragen beschäftigt sich intensiv der Intergovernmental Panel on Climate Change (IPCC) und im ersten Abschnitt des ersten Kapitels des Buches wird versucht, die ca. 2700 Seiten des dritten Assessmentreports des IPCC (TATuP 3/2001, S. 93 ff.) auf etwa 9 Seiten zusammenzufassen. Da es sich hier um eine reine Pflichtübung handelt, die zum Rest des Buches wenig beiträgt, sei nur die Warnung ausgesprochen, dass man sich mit dem Versuch, das Klimasystem mit dem gesunden Menschenverstand und einigen illustrativen Zahlen zu beschreiben, auf das Niveau vieler Klimaskeptiker begibt, die mit ihren ,handgemachten" Klimatabellen bezweifeln, dass es überhaupt einen Klimawandel gibt. Schon 1955 wies John von Neumann darauf hin ,that the problem of forecasting long-term fluctuations of the general circulation will involve a great deal of mathematical analysis and a great deal of calculation. It will not be possible to arrive at the answers by dialectic methods". (von Neumann 1960, S. 11) Dies wird übrigens anhand des Beispiels verdeutlicht, dass es unmöglich sei, ad hoc von der Änderung der die Erde erreichenden Sonneneinstrahlung auf die resultierende Klimaänderung, Erwärmung oder Abkühlung zu schließen.

Der zweite Abschnitt des ersten Kapitels beschäftigt sich mit der Vorgeschichte und dem Inhalt der Klimarahmenkonvention und des Kyoto-Protokolls. Ganz allgemein dienen solche Protokolle (man denke insbesondere auch an das Montreal-Protokoll) der ,konkreten und substantiellen Ausfüllung der Rahmenkonvention". In einer Tabelle werden die Artikel von Klimarahmenkonvention und Kyoto-Protokoll gegenübergestellt. Zusammen mit den dazugehörigen Fußnoten ergibt sich ein schneller Überblick über die Themenbereiche und die entsprechenden Artikel. Für alle, die nicht täglich im Kyoto-Protokoll lesen, eine gute Gedächtnisstütze. Ansonsten enthält dieser $\mathrm{Ab}$ schnitt nach einer Einordnung des Protokolls in das „völkerrechtliche Klimaregime“ auf etwa 30 Seiten eine vollständige, aber kompakte Zusammenfassung des Protokollinhalts.

\section{Umweltvölkerrechtliche Nichteinhaltungsverfahren}

Im dritten Kapitel geht es nun wirklich in medias res und um eine vergleichende Darstellung von Nichteinhaltungsverfahren im Umweltvölkerrecht, um die langwierigen Verhandlungen zur Ausgestaltung des Nichteinhaltungsverfahrens des Kyoto-Protokolls im Speziellen und um seine Rechtsform.

Das erste umweltvölkerrechtliche Nichteinhaltungsverfahren ist das des Montrealer Protokolls und lag schon 1990 in einer ersten Fassung vor, weitere aufgeführte Verträge mit den dazugehörigen Protokollen sind die Genfer Konvention über weiträumige grenzüberschreitende Luftverunreinigungen (LRTAP), die Aarhus Konvention über den Zugang zu Informationen, die Alpenkonvention und andere, deren Nichteinhaltungsverfahren zum Teil schon vorliegen. Sicher auch sehr interessant wäre ein Blick über den Tellerrand auf andere völkerrechtliche Abkommen gewesen. Hier hat man es oft mit ähnlichen Problemen zu tun, die einerseits die Einhaltung der Abmachungen betreffen und auf der anderen Seite auch deren Durchsetzung. So ist der Vertrag über das umfassende Verbot von Atomtests (CTBTO) mit einem umfangreichen, auf einem weltumspannenden Netz von Überwachungsstationen basierenden Verifikations-Regime ausgestattet. Inwieweit nach einem Inkrafttreten dieses Vertrages der Druck auf Staaten, sich an diesen Vertrag zu halten, aus seinen Organen selbst kommt oder aber im Grunde nur von aktuellen Machtkonstellationen abhängt, könnte durchaus auch Hinweise darauf geben, wie in Zukunft die Einhaltung emissionsmindernder Maßnahmen oder der Finanztransfer hin zu ärmeren und vom Klimawandel besonders betroffenen Ländern durchgesetzt werden könnten. ${ }^{1}$

Aber zurück zum hier zu besprechenden Buch. Im Anschluss an die Einordnung des Nichteinhaltungsverfahrens des Kyoto-Protokolls werden die Etappen der Verhandlungen dazu seit 1998 (COP 4 in Buenos Aires) bis 2001 (COP 7 in Marrakesch) beschrieben. Auch wenn die sehr ausführliche und zudem noch bebilderte Beschreibung zunächst nur an ein Insider-Publikum gerichtet zu sein scheint, gibt sie doch einen Blick auf den Verhandlungsmarathon mit all seinen Gruppierungen und Allian- 
zen wie JUSSCANNZ, G77, „Umbrella Group“ etc. frei. ${ }^{2}$ Außerdem werden die Probleme und Winkelzüge bei der Formulierung der Texte zum Nichteinhaltungsverfahren thematisiert.

Nicht nur gegenüber den „Kapriolen“ des Klimas, sondern auch im Hinblick auf die Verhandlungen, scheint einem häufig der so genannte gesunde Menschenverstand zu versagen. So beispielsweise, wenn auf der Vertragsstaatenkonferenz in Marrakesch nur der Text des Nichteinhaltungsverfahrens angenommen wird, nicht aber das Verfahren selbst, oder man in einem „non-amendment amendment“ übereinstimmt, nicht übereinzustimmen. Der juristisch vorgebildete Beobachter hingegen erklärt, auch hierbei handele es sich um einen Fortschritt in den Verhandlungen, man habe das Problem eben „bewusst offen gelassen“. Andererseits seien auch künftig noch Differenzen zu erwarten, weil das Problem der Rechtsnatur des Nichteinhaltungsverfahrens nur vertagt worden sei (S. 109). ${ }^{3}$ Der kurze Artikel 18 im KyotoProtokoll zum Nichteinhaltungsverfahren ist zwar völkerrechtlich verbindlich, hat aber nur eine „Platzhalterfunktion“ und schränkt Beschlüsse zu verbindlichen Folgen eines solchen Verfahrens kaum ein (S. 111).

\section{Analyse und Folgen des Nichteinhaltungsverfahrens}

In den nächsten drei Kapiteln des Buches geht es nun um den vorliegenden und im Anhang abgedruckten Text des Nichteinhaltungsverfahrens selbst; um den Einhaltungsausschuss, um die Verfahrensregeln und um die ,angedrohten" Folgen des Nichteinhaltungsverfahrens. Um sich einen ersten Überblick über die in den einzelnen Kapiteln abgehandelten Probleme zu verschaffen, ist gerade hier ein Blick in die übrigens auch den anderen Kapiteln des Buches jeweils angehängten - ,Zwischenergebnisse" in jedem Falle sinnvoll. Genaueres Lesen könnte aber durchaus spannend werden, wenn der Einhaltungsausschuss einmal aktiv geworden sein sollte, wenn offensichtlich wird, ob und wie die Verfahrensregeln greifen, zu welchen Folgen das Nichteinhaltungsverfahren tatsächlich führen wird und wie über all dies gestritten werden wird. Im Einzelnen betrifft das Nichteinhaltungsverfahren nicht nur das generelle Reduktionsziel des Kyoto-Protokolls, sondern auch die so genannten methodischen oder Berichtspflichten und die Berechtigungsvoraussetzungen für die flexiblen Mechanismen wie Clean Development Mechanism, Joint Implementation oder den Emissionshandel.

Um welche Folgen geht es hier eigentlich? Sie werden in der hier besprochenen Dissertation eingehend beschrieben, ökonomisch analysiert und diskutiert. Beim Lesen stellt sich jedoch immer wieder das oben schon erwähnte Bild der Loriotschen Skatrunde ein. Zunächst einmal kommt bei einem Verstoß gegen die methodischen oder Berichts-Pflichten (insbesondere die regelmäßige Erstellung nationaler Emissionsinventare) unterstützend ein Einhaltungsaktionsplan zum Tragen. Schon dadurch wird die Nichteinhaltung öffentlich dokumentiert, was zu einem Renommeeverlust der Vertragspartei führen kann. Bei Nichteinhaltung der Emissionsverpflichtungen sind zusätzlich Sanktionen vorgesehen, wie zum Beispiel die Reduktion der in einem weiteren Kyoto-Verpflichtungszeitraum zugeteilten Menge an Emissionsberechtigungen durch die überschrittene Menge multipliziert mit einen Straffaktor. Ob es allerdings eine weitere Kyoto-Periode geben wird und welche Emissionsminderungen für diese beschlossen werden wird, steht noch gar nicht fest. Auch angesichts der derzeit für den Zeitraum bis 2020 diskutierten Emissionsminderungen von bis zu $40 \%$ gegenüber 1990 sind solche Sanktionen eher „peanuts“. Falls es zu weiteren Kyoto-Perioden kommen sollte, besteht für die betreffenden Parteien darüber hinaus immer noch die Möglichkeit, für die nächste Verpflichtungsperiode eine entsprechend hohe Zuteilung herauszuhandeln, so dass der Straf-Faktor gar nicht greifen würde, die Tilgung auf weitere Verpflichtungsperioden hinauszuschieben oder ganz aus dem Kyoto-Protokoll auszusteigen.

Der Autor betont allerdings, dass eben diese Schlupflöcher es erlaubt hätten, dass das Nichteinhaltungsverfahren von zweifelnden Parteien akzeptiert und ratifiziert wurde. Im Grunde führt all dies in der abschließenden Beurteilung und Bewertung durch Einhaltungstheorien ohne Umwege zum „EinhaltungsRätsel“", dass nämlich in der Regel Völkerrecht eingehalten wird (S. 266 f.). Dass dies auch für das Kyoto-Protokoll aufgrund seines aus theoretischer Sicht gelungenen Nichteinhaltungsverfahrens so sein könnte, wird vom Autor 
positiv beantwortet. Trotzdem sei nicht abschließend feststellbar, ob letzteres sein Ziel in der Praxis erreichen könne. Es wird ein Satz zitiert, der an das Zitat von Neumanns zur Klimaforschung oben erinnert: „Der Komplexitätsgrad des sozio-politischen Raums, in dem das Völkerrecht seine verhaltensnormierende Funktion ausübt, rührt an die Grenze der wissenschaftlichen Erkenntnisfähigkeit" (S. 310). Trotz all dieser Unsicherheiten (oder wegen), kommt der Autor zum Schluss, dass ,das Nichteinhaltungsverfahren nicht der einzige und wahrscheinlich nicht einmal der maßgebliche Faktor sei, der über Einhaltung oder Nichteinhaltung entscheiden wird“ (S. 311).

\section{Abschließende Bemerkung}

Beim Lesen dieses Buches tritt häufig ein zwiespältiges Gefühl auf: Auf der einen Seite die geradezu hermetische Analyse und Durchdringung des Textes des Nichteinhaltungsverfahrens durch den Autor und auf der anderen Seite die Unwägbarkeiten des Klimaregimes, die natürlich ebenfalls thematisiert werden, allerdings auch in einem ganz anderen Stil als in den mehr juristisch orientierten Textteilen. Das erinnert ein wenig an den Gegensatz zwischen dem eingespielten Verhandlungsmarathon zur Klimarahmenkonvention mit seinen vielen hundert, von Konferenz zu Konferenz in geschlossenen, abgedunkelten und klimatisierten Sälen, reisenden Teilnehmern, während draußen der Klimawandel schon längst stattfindet.

\section{Anmerkungen}

1) Dass hier auch Sicherheitsaspekte zum Tragen kommen, haben vor einiger Zeit die mehrfachen Warnungen amerikanischer Militärs und die Sitzung des UN-Sicherheitsrates zum gleichen Thema im Frühjahr dieses Jahres deutlich gezeigt. Auch der WBGU hat im Juni ein Gutachten mit dem Titel ,Sicherheitsrisiko Klimawandel" herausgegeben (WBGU 2007).

2) JUSSCANNZ: Japan, USA, Switzerland, Canada, Australia, Norway, New Zealand; G77: Gruppe der 77 (Zusammenschluss von derzeit 130 Staaten der Dritten Welt); Umbrella Group: Eine lose Koalition von nicht EU-Staaten, teilweise deckungsgleich mit den JUSSCANNZStaaten plus Russland.
3) Die Dissertation selbst befindet sich übrigens auf dem Stand vor der ersten Sitzung der Vertragsparteien des Kyoto-Protokolls (COP/MOP 1) in Montreal 2005, wo das Nichteinhaltungsverfahren zwar angenommen wurde, man aber die Frage zur Rechtsnatur des Verfahrens - insbesondere inwieweit man ihm durch eine Änderung des Kyoto-Protokolls völkerrechtliche Verbindlichkeit gibt - weiter auf Ende 2007, der Konferenz in Bali, verschoben hat.

\section{Literatur}

Neumann, J. von, 1960: Some Remarks on the Problem of Forecasting Climate Fluctuations. In: Pfeffer, R.L.: Dynamics of Climate. Oxford, pp. 9-11

Holtwisch, Chr., 2007: Asiatisch-pazifische Partnerschaft für umweltverträgliche Entwicklung und Klima - Blockade oder Antrieb für das internationale Klimaregime? Interdisziplinäres Fernstudium Umweltwissenschaften (infernum), Masterarbeit, Vreden

WBGU - Wissenschaftlicher Beirat der Bundesregierung Globale Umweltveränderungen, 2007: Welt im Wandel: Sicherheitsrisiko Klimawandel. Berlin; http://www.wbgu.de/wbgu_jg2007.pdf

\section{Vom Mittel zum Medium Zum Wandel des Technik- verständnisses}

\section{Chr. Hubig: Die Kunst des Möglichen I - Technikphilosophie als Reflexion der Medialität. Bielefeld: Transcript Verlag 2006, 270 S., ISBN 3-89942-431, € 24,80}

\section{Rezension von Jan C. Schmidt, Georgia Institute of Technology, Atlanta, USA}

\section{Verlust an Spuren}

„Die Hochtechnologien zeitigen einen neuen Verlust der Spuren“, so der Technik- und Kulturphilosoph Christoph Hubig in seinem neuesten, für die TA-Community anregenden Buch „Die Kunst des Möglichen I. Technikphilosophie als Reflexion der Medialität.“ 